Artículo

\title{
Redes de confianza y riesgo agroclimático en la asistencia técnica agropecuaria, Colombia
}

\author{
Lilia Constanza Molano-Bernal ${ }^{1}$ \\ Julia Sánchez-Gómez ${ }^{2 \S}$ \\ Juan Carlos Martínez-Medrano ${ }^{1}$ \\ Marisol Vázquez-Alfaro ${ }^{3}$
}

${ }^{1}$ Corporación Colombiana de Investigación Agropecuaria (Agrosavia). Vía Mosquera-Bogotá km 14, Colombia. CP. 250047. (lcmolano@agrosavia.co; jcmartinezm@agrosavia.co). ${ }^{2}$ Cátedra CONACYTCentro de Investigación y Asistencia en Tecnología y Diseño del Estado de Jalisco, AC (CIATEJ). Av. de los Normalistas 800, Colinas de la Normal. Guadalajara, Jalisco, México. CP. 44270. ${ }^{3}$ INIFAP. Carretera Libre Tepatitlán-Lagos de Moreno km 8, Tepatitlán de Morelos, Jalisco, México. CP. 47600. vazquez.alfaro.marisol@gmail.com.

${ }^{\S}$ Autor para correspondencia: jsanchez@ ciatej.mx.

\section{Resumen}

El objetivo de la presente investigación fue analizar los cambios en las redes de confianza técnica de los Asistentes Técnicos Agropecuarios (ATA), después de un proceso de gestión y difusión de una cultura del riesgo agroclimático durante un periodo de dos años en los departamentos Cundinamarca, La Guajira y Valle del Cauca, Colombia. Se realizaron entrevistas a los ATA, en dos momentos de observación: al inicio (2015) y al finalizar la intervención del proyecto modelos de adaptación y prevención agroclimática (2017). El análisis de redes sociales se utilizó como herramienta de análisis y de intervención, esta permitió identificar la estructura de la red y los actores con mayor cobertura de difusión de la información en los departamentos. La intervención del proyecto MAPA provocó cambios estructurales en las redes de confianza técnica de los ATA y su percepción sobre la importancia del tema del riesgo agroclimático en la prestación de los servicios de extensión. En Cundinamarca y La Guajira los ATA disminuyeron el tamaño de su red ( $p<0.05)$, sin embargo, el indicador de intermediación aumentó de forma significativa $(p<0.05)$. Se concluye que, al intervenir en las redes de confianza de los asistentes técnicos agropecuarios, mediante el diseño de una estrategia comunicativa y la identificación de actores clave para difundir el tema de riesgo agroclimático; se favoreció a una mayor vinculación, el acceso, intercambio y alcance de la información.

Palabras clave: adaptación al cambio climático, difusión de la información, gestión del conocimiento.

Recibido: febrero de 2021

Aceptado: abril de 2021 


\section{Introducción}

La alteración de los patrones climáticos afecta indudablemente a los sistemas de producción agropecuaria (Vergara et al., 2014). En Colombia, el fenómeno climático de La Niña durante el año 2010, provocó pérdidas en el sector agropecuario que ascendieron a 387.5 millones de pesos en el caso de cultivos transitorios, para los cultivos permanentes las pérdidas se estimaron en 306 millones de pesos, en la ganadería fueron 24 millones de pesos en la producción de carne y el acopio de leche cayó entre el 25 y 30\%, la reducción se valoró en 400 L diarios no recogidos y en la avicultura las pérdidas fueron de 2.4 millones de pesos incluyendo el valor de las aves muertas (CEPAL, 2012).

Además, en los escenarios climáticos para 2030 indican un incremento general de la temperatura en todo el país (con mayor intensidad en el sur), lo que podría causar escasez de agua para la producción agrícola (CIMMYT, 2019). En ese sentido, en el año 2010 se creó el Fondo Adaptación en Colombia, con el objetivo de impulsar soluciones para los afectados directos por el fenómeno climático de La Niña y brindar respuestas de adaptación a fenómenos climáticos extremos en el país. En el mismo contexto, la Corporación Colombiana de Investigación Agropecuaria (Agrosavia), tuvo la iniciativa de formular el proyecto modelos de adaptación y prevención agroclimática (MAPA), con el propósito de contribuir al desarrollo de las capacidades locales del subsistema de asistencia técnica agropecuaria (SSATA) para la difusión de una cultura de adaptación al cambio climático y la variabilidad climática.

Esto bajo la premisa, de que las redes son espacios donde se intercambian, generan y comparten conocimientos como motor de desarrollo y competitividad (Pérez y Castañeda, 2009). Además, el trabajo en red puede contribuir a facilitar el acceso a recursos, el acceso a nuevas ideas y acelerar la transferencia del conocimiento (Powell and Grodal, 2006).

El análisis de redes es una herramienta que ha sido utilizada en diferentes ámbitos, como por ejemplo la economía, mercadeo, salud y por supuesto en el sector agropecuario, en este último se ha empleado para entender los procesos de adopción y difusión de tecnologías entre los productores y gestionar la innovación (Monge y Hartwich, 2008; Aguilar-Gallegos et al., 2016). En el tema de riesgo agroclimático las redes podrían tener gran potencial, ya que estas ofrecen dos recursos valiosos: flujos de conocimiento y acción a gran escala. Sin embargo, el reto es identificar la estrategia adecuada para hacer el mejor uso de las redes y medir el impacto tangible de la gestión (Schwaab y Seibold, 2014).

En el proyecto MAPA, se utilizó el análisis de redes sociales (ARS) con el fin de optimizar la circulación de la información sobre el tema del riesgo agroclimático (mensaje técnico) entre los asistentes técnicos agropecuarios que conforman los subsistemas de asistencia técnica agropecuaria de 18 departamentos en Colombia. En este sentido, el objetivo de la presente investigación fue analizar los cambios en las redes de confianza técnica de los asistentes técnicos agropecuarios (ATA), después de un proceso de gestión y difusión de una cultura del riesgo agroclimático durante un periodo de dos años en los departamentos Cundinamarca, La Guajira y Valle del Cauca, Colombia. 


\section{Materiales y métodos}

El proyecto modelos de adaptación y prevención agroclimática (MAPA) se desarrolló en 54 municipios de 18 departamentos de Colombia: Atlántico, Bolívar, Boyacá, Cauca, Cesar, Chocó, Córdoba, Cundinamarca, Huila, La Guajira, Magdalena, Nariño, Norte de Santander, Santander, Sucre, Tolima y Valle del Cauca. En los que se consideraron 54 sistemas productivos ganaderos y agrícolas como prioritarios: ají topito, ganadería doble propósito, plátano, cacao, caña panelera, lulo, maíz blanco, yuca industrial, arroz secano, entre otros.

En el presente estudio se tomaron como muestra del proceso de gestión del proyecto MAPA tres departamentos representativos de Colombia: Cundinamarca, La Guajira y Valle del Cauca (Figura 1), de los cuales se contaba con la información sistematizada al inicio del proyecto en 2015 y al finalizar el mismo en 2017.

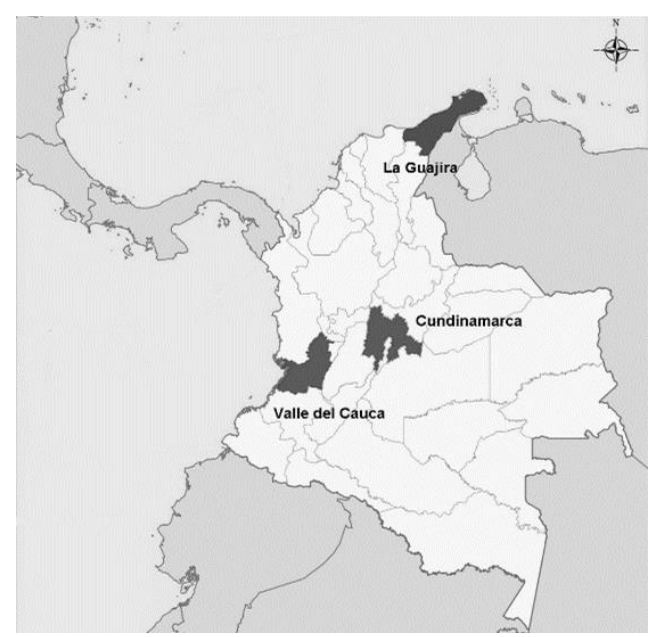

Figura 1. Localización geográfica de Cundinamarca, La Guajira y Valle del Cauca en Colombia.

Para el estudio se diseñaron y aplicaron dos encuestas, la primera sirvió para realizar la caracterización del subsistema de asistencia técnica agropecuaria (SSATA) departamentales y analizar la percepción de los asistentes técnicos agropecuarios (ATA) con respecto el riesgo agroclimático. La encuesta se organizó en tres bloques: 1) información del asistente técnico, como su edad, experiencia, escolaridad, entre otros; 2) redes de confianza técnica e información sobre la prestación del servicio de asistencia técnica, donde se solicitó organizar en orden de importancia las herramientas que consideran necesarias para prestar el servicio de asistencia técnica; y 3) conocimientos sobre el riesgo agroclimático, percepción y variabilidad climática.

Una vez identificados los diferentes actores prestadores del servicio de asistencia técnica en cada territorio, se realizó la segunda encuesta para analizar los vínculos de confianza técnica de los ATA y elaborar el sociograma de la red, la pregunta base fue: ¿a quién de sus colegas consulta cuando tienen una duda técnica? Esto permitió indagar sobre aquellas personas a quienes recurren para consultar aspectos técnicos del sistema productivo u otras dudas relacionadas con su desempeño como asistente técnico. Con la información obtenida se construyó el gráfico de la red de confianza técnica, en el que se evidenciaron las relaciones existentes entre los ATA, ya identificados en la caracterización. 
La encuesta de caracterización y percepción, así como, la de relacionamiento se aplicó a los ATA de cada departamento en dos momentos de observación, al inicio del proyecto enero 2015 y al finalizar, en enero de 2017. En el caso de los departamentos analizados en esta investigación, al inicio del proyecto la encuesta de caracterización y percepción fue contestada por 217 asistentes técnicos, los cuales fueron identificados en campo mediante la técnica de bola de nieve. En el año 2017 se levantó una encuesta similar a la del año 2015 pero través de una plataforma virtual, se obtuvo respuesta de diez asistentes técnicos agropecuarios de Cundinamarca, siete de La Guajira y once del Valle del Cauca. El número de encuestas de relacionamiento se detalla en el Cuadro 1.

Cuadro 1. Número de ATA que respondieron la encuesta de relación por departamento analizado.

\begin{tabular}{ccc}
\hline Departamento & $\begin{array}{c}\text { Núm. de actores } \\
\text { (enero de 2015) }\end{array}$ & $\begin{array}{c}\text { Núm. de actores } \\
\text { (mayo de 2017) }\end{array}$ \\
\hline Cundinamarca & 58 & 30 \\
La Guajira & 41 & 29 \\
Valle del Cauca & 41 & 53 \\
\hline
\end{tabular}

Encuestas aplicadas a los ATA del proyecto MAPA, 2015 y 2017.

Con la información obtenida de las encuestas, se construyó una base de datos en Excel y un archivo de bloc de notas con los vínculos entre los asistentes técnicos agropecuarios. A cada uno se le asignó un identificador y un número consecutivo para el fácil manejo de los datos en los sociogramas de las redes. Para la elaboración de los gráficos y cálculo de los indicadores de las redes se utilizó el software Ucinet versión 6.628 para Windows.

Los indicadores de la red calculados fueron: la densidad, que refiere al porcentaje de relaciones existentes entre las posibles, y el índice de centralización, que señala que tan concentrados se encuentran los vínculos por un actor o grupo de actores. Los indicadores del ego o nodo utilizados fueron: tamaño de la red nodal, que refiere al número de actores con los que se encuentra directamente conectado; e intermediación que muestra el número de pares no conectados directamente en la red del actor, dividido entre el total de pares existentes.

El cálculo de la cobertura de difusión y estructuración de cada actor se realizó con el programa Keplayer $2^{\odot}$. Se utilizaron los algoritmos de harvest, diffuse y disrupt para identificar a los principales ATA que podrían tener acceso a la información (actores colectores), influenciar a otros a través de sus vínculos (actores fuente) o causar la fragmentación de red (actores articuladores), de acuerdo con el grado nodal y su cercanía en la red (Borgatti, 2006).

Para medir los cambios en los indicadores del ego o nodo de las redes de confianza técnica de los asistentes técnicos agropecuarios al inicio y final del proyecto, se utilizó una prueba de ' $t$ ' en muestras independientes. Esta prueba permite observar si la diferencia en las medias de los indicadores es estadísticamente significativa. El análisis estadístico de los datos se realizó en el paquete Statistical Analysis Software (SAS) v.9. Estos indicadores y el análisis gráfico de las redes fueron útiles para medir los cambios generados por el efecto de la intervención del proyecto modelos de adaptación y prevención agroclimática (MAPA). 


\section{Resultados y discusión}

\section{Caracterización de los asistentes técnicos agropecuarios}

En el departamento de Cundinamarca la figura institucional predominante para la prestación del servicio de asistencia técnica son las unidades municipales de asistencia técnica agropecuaria (UMATA), las Secretarías de Desarrollo Económico (SDE) y las Secretarías de Agricultura Municipales (SAM). En Cundinamarca, 105 de 116 municipios funcionan bajo esta modalidad, el tipo de contratación de los asistentes técnicos es anual y en algunas ocasiones por período de cuatro años, lo que corresponde al período de elección de los alcaldes municipales.

Por otro lado, cuando la asistencia técnica es brindada por entidades prestadoras del servicio de asistencia técnica agropecuaria (EPSAGRO), la vinculación laboral se realiza mediante contratos a término fijo con una duración no mayor a diez meses. Este tipo de contratación por cortos periodos afecta directamente la continuidad del personal en las zonas de trabajo, existe alta rotación y movilidad de los ATA, la permanencia de los actores en los territorios e incluso de las entidades no está garantizada y genera inestabilidad laboral (Méndez, 2015). Lo cual afecta, la continuidad de procesos de intervención y los procesos de desarrollo de capacidades (Corpoica, 2105a; Monsalvo et al., 2017)

A diferencia, en el Valle del Cauca, existen diferentes entidades que prestan el servicio de asistencia técnica, como el Centro Provincial de Gestión Agroempresarial del Sur Occidente conformado por los municipios de: Palmira, Candelaria, Florida, Guacarí, Ginebra, Pradera y El Cerrito, que trabajan de la mano con las secretarías de agricultura de estos municipios (Corpoica, 2015b). El desarrollo institucional en este departamento es significativo, la presencia de gremios productivos en el departamento es relevante, tal es el caso de la Asociación Hortofrutícola de Colombia (Asohofrucol), la Federación Nacional de Cafeteros y la Asociación de Cultivadores de Caña de Azúcar de Colombia (ASOCAÑA) que prestan asistencia técnica a sus asociados.

En los tres departamentos analizados en este trabajo, el servicio de asistencia técnica en su mayoría es ofrecido por hombres con una edad alrededor de los 40 años, y con una experiencia promedio en la actividad menor a los 10 años (Cuadro 2). En relación con la formación académica de los ATA, el 39.0\% tienen formación profesional en áreas agropecuarias como la ingeniería agronómica, medicina veterinaria y zootecnia. Similar a lo reportado en otros estudios donde se ha encontrado que los servicios de extensión son brindados principalmente por hombres y con formación a fin a ciencias agronómicas y zootécnicas, dejando de lado a las ciencias sociales (Landini, 2013; Mayoral-García et al., 2015; Monsalvo et al., 2017).

En Cundinamarca $61 \%$ de los ATA presentaron un nivel educativo de técnico y tecnólogo, según la Ley 30 de 1992, por la cual se organiza el servicio público de la Educación Superior en Colombia, la educación técnica es aquella que ofrece programas de formación en ocupaciones de carácter operativo e instrumental y de especialización en su respectivo campo de acción, sin perjuicio de los aspectos humanísticos propios de este nivel y la educación tecnológica ofrece programas de formación en ocupaciones, programas de formación académica en profesiones o disciplinas y programas de especialización, por el contrario, en el Valle del Cauca se observó un mayor número de especialistas. 
Cuadro 2. Características de los asistentes técnicos en los departamentos analizados.

\begin{tabular}{ccccc}
\hline Variable & & Cundinamarca & La Guajira & Valle del Cauca \\
\hline Edad (años) & & 36 & 43 & 42 \\
Hombre\% & & 75.5 & 88.8 & 71.1 \\
Mujeres\% & & 24.5 & 11.2 & 28.9 \\
Experiencia como ATA(\%) & $1-5$ años & 42.5 & 38.1 & 44.2 \\
& 6-10 años & 20.3 & 26.3 & 7.7 \\
& $11-16$ años & 9.3 & 13.6 & 13.5 \\
Nivel educativo (\%) & $>16$ años & 27.7 & 19 & 34.6 \\
& Técnico & 42.5 & 31.8 & 17.3 \\
& Tecnólogo & 18.5 & 13.6 & 19.2 \\
& Profesional & 31.4 & 41.8 & 42.3 \\
& Especialista & 9.3 & 11.8 & 21.2 \\
\hline
\end{tabular}

Encuestas aplicadas a los ATA del proyecto MAPA, 2015.

\section{Percepción de asistentes técnicos agropecuarios sobre gestión del riesgo agroclimático}

Al iniciar el proceso de difusión de la información en el marco del proyecto modelos de adaptación y prevención agroclimática (MAPA), se observó que los ATA de Cundinamarca, La Guajira y Valle del Cauca percibían la gestión del riesgo agroclimático como una herramienta o tema poco relevante para prestar un adecuado servicio de asistencia técnica, alrededor de una tercera parte (27\%) lo consideraba un tema poco o nada prioritario. Esto podría explicarse porque los ATA de Colombia como los de otros países, otorgan mayor relevancia o se enfocan a temas principalmente técnicos, sin embargo, el aspecto social, económico y ambiental también es tarea de los agentes de desarrollo rural del país (Sáenz-Torres, 2014).

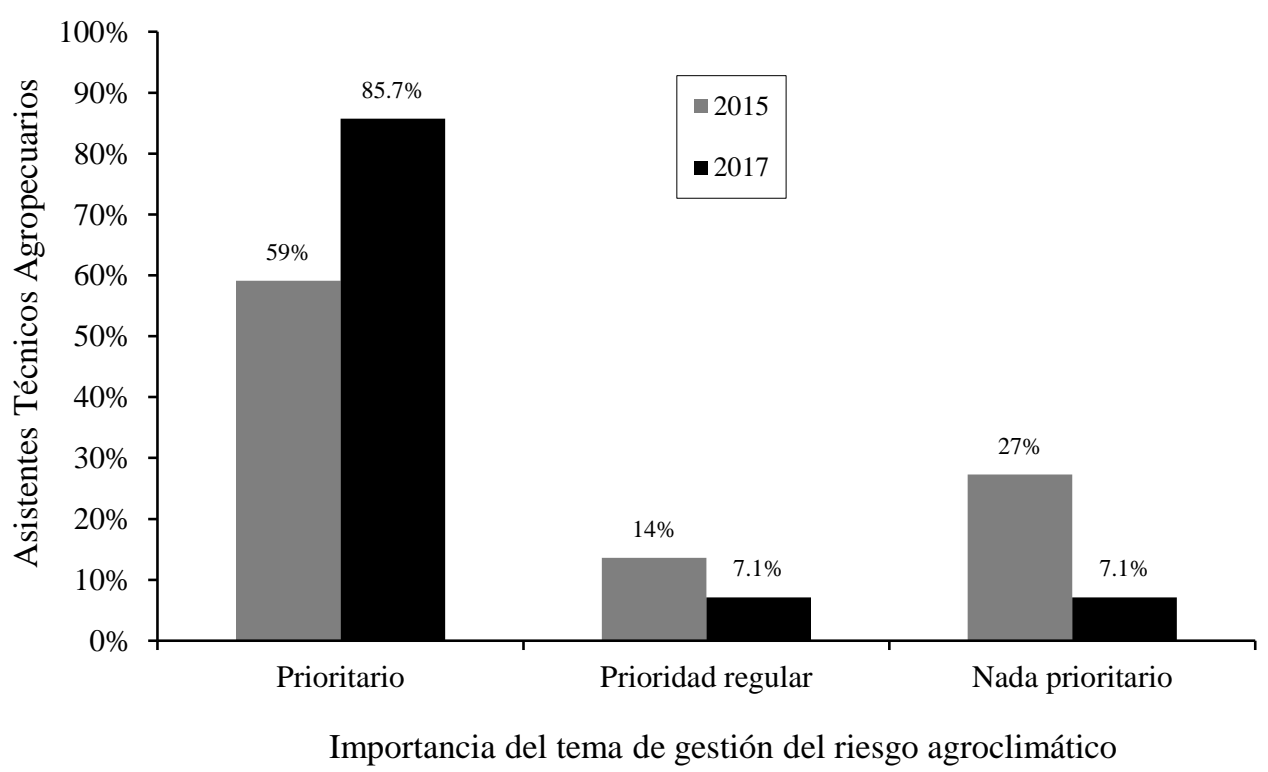

Figura 2. Importancia otorgada por los ATA al tema de gestión del riesgo agroclimático. Encuestas aplicadas a los ATA del proyecto MAPA, 2015 y 2017. 
El proyecto MAPA estructuró una estrategia de capacitación denominada momentos formativos que se desarrolló de la siguiente manera: 1) análisis de los conceptos de cambio climático; 2) gestión de la información agroclimática (datos de clima, variables climáticas y cómo se interpretan); 3) sistema experto y sistemas de alerta temprana (como tomar decisiones basadas en información); 4) gestión del riesgo agroclimático (valoración del riesgo agroclimático en los sistemas productivos); y 5) opciones tecnológicas de los cultivos definidos (valorar tecnologías para implementarse en los sistemas productivos y poder disminuir el riesgo agroclimático.

Para su difusión se conformaron grupos de WhatsApp donde se generó una rutina comunicativa semanal y se identificaron actores clave para difundir temas específicos. Talleres presenciales prácticos y participativos cada cuatro meses con los asistentes técnicos. Una vez finalizada la estrategia de capacitación del proyecto MAPA en el año 2017, la información climática fue reconocida por $85.7 \%$ de los ATA como una herramienta prioritaria o necesaria en la asistencia técnica, cabe mencionar que su asistencia era por decisión propia y financiada con recursos propios.

\section{Redes de confianza técnica de los asistentes técnicos agropecuarios}

En los departamentos de Cundinamarca y La Guajira se constituyeron redes en el año 2015 con 58 y 41 actores respectivamente, y estas redes al final del proceso fueron de 30 y 29 actores (Cuadro 3). Al respecto Zarazúa-Escobar et al. (2012) aportan evidencia empírica sobre el proceso evolutivo e integración a las redes, donde aquellas que involucran colaboración tienden a reducirse en relación con las que sólo se integran por un nivel de reconocimiento entre los actores.

Cuadro 3. Indicadores de las redes de confianza técnica de los ATA, 2015 y 2017.

\begin{tabular}{ccccccccc}
\hline \multirow{2}{*}{ Indicador } & \multicolumn{2}{c}{ Cundinamarca } & & \multicolumn{2}{c}{ La Guajira } & & \multicolumn{2}{c}{ Valle del Cauca } \\
\cline { 2 - 3 } & 2015 & 2017 & & 2015 & 2017 & & 2015 & 2017 \\
\hline Tamaño de la red & 58 & 30 & & 41 & 29 & & 41 & 53 \\
Número de vínculos & 630 & 148 & & 617 & 94 & & 117 & 304 \\
Centralización de entrada (\%) & 23.4 & 25.3 & & 35.7 & 25 & & 15.8 & 63.2 \\
Centralización de salida (\%) & 27 & 28.8 & & 38.3 & 28.7 & & 8.1 & 20.1 \\
Densidad (\%) & 19.1 & 17 & & 37.6 & 11.6 & & 7.1 & 11 \\
\hline
\end{tabular}

Encuestas aplicadas a los ATA del proyecto MAPA, 2015 y 2017.

En las dos redes también el índice de centralización de salida fue ligeramente mayor que la de entrada, lo que mostró la existencia de un grupo de asesores técnicos agropecuarios colectores de información y con inquietud de adquirir nuevos conocimientos por medio de sus vínculos, alcanzando a obtener información de alrededor de una tercera parte o más de los miembros que integran las redes.

Por otra parte, la red del Valle de Cauca mostró una tendencia diferente, aumentó el número de nodos en la red y sus vínculos estuvieron cerca de triplicarse, lo que se reflejó en un incremento de la densidad de relaciones 3.9\%. En ocasiones el tamaño de las redes se incrementa, debido a las relaciones de un actor enviadas hacia la red, proveen acceso a varios y diversos nodos, permitiéndole alcanzarlos y además insertarse en la red (Aguilar-Gallegos et al., 2016). 
Los incrementos en la densidad están relacionados con mayores niveles de homogeneidad en el comportamiento de los individuos (Monge y Hartwich, 2008), por lo que en este caso la densidad se reflejó en mayor intercambio de información y por ende, de aprendizaje. Así también, el índice de centralización de entrada se incrementó en la red con $63.2 \%$, por la existencia de actores con una posición central, y reconocidos por sus pares como fuentes de información. Los niveles de centralización de entrada aumentan de manera positiva cuando existe un número reducido de actores reconocidos como fuentes de información (Aguilar-Gallegos et al., 2017).

En este caso, suministraban información del tema de riesgo agroclimático a más de la mitad de los actores en la red en el año 2017. El análisis de redes sociales (ARS) es útil para analizar los patrones de interacción entre agricultores (o técnicos) y actores presentes en una red, lo que permite entender los procesos de búsqueda de información y conocimiento (Aguilar-Gallegos et al., 2016). La herramienta en el presente estudio permitió identificar a los ATA con una posición estratégica en la red, que dada su buena reputación técnica garantizarían la circulación del mensaje técnico sobre el riesgo agroclimático y con una mayor cobertura entre los miembros de las redes.

La cobertura de estos actores es relevante, en el sentido de que su alcance no se limita sólo a los vínculos directos, sino que, de permanecer en el territorio podrían tener un alcance mayor en el proceso de enseñanza con otros técnicos de la red o de la región (López et al., 2016).

\section{Cundinamarca}

La red de Cundinamarca en el año 2015 se observó estructurada y con alta conectividad, dos actores identificados con los códigos AT343 y AT349 eran las principales fuentes de información, y a su vez, colectores de información en la red con una cobertura alcanzada $96.4 \%$ en sus dos roles, por lo que fueron idóneos para transmitir el mensaje técnico sobre riesgo agroclimático (Figura 3).
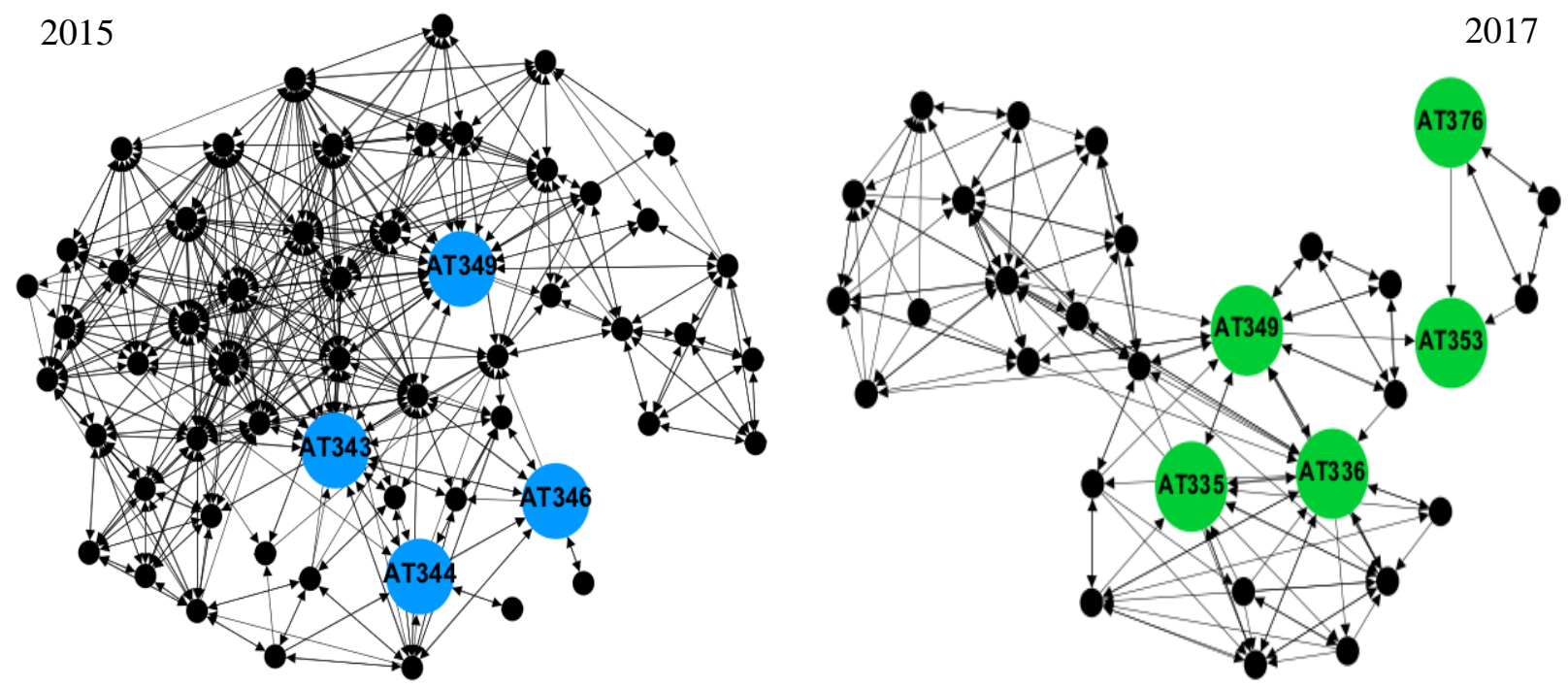

Figura 3. Red de confianza técnica de los ATA de Cundinamarca 2015 y 2017. Encuestas aplicadas a los ATA del proyecto MAPA, 2015 y 2017. 
El primero era parte de entidades prestadoras del servicio de asistencia técnica agropecuaria (EPSAGRO)-Asociación Hortifrutícola de Colombia (ASOHOFRUCOL) en el municipio de Útica, y el segundo, de la unidad municipal de asistencia técnica agropecuaria (UMATA) del municipio de Ubaté.

Por otra parte, debido a la estructura densa de la red, esta no dependía de actores articuladores o enlaces que pudieran ocasionar una ruptura en la red por su salida, el AT344 un técnico independiente de Caparrapí y el AT346 de la UMATA del municipio Quebradanegra, mostraron el mayor índice de fragmentación con sólo13\%. Sin embargo, las redes no son estáticas y tienden a mostrar dinámicas temporales en función de la etapa del proceso de gestión (Bodin et al., 2017) y la red final mostró menor grado de conectividad, que su contraparte del año 2015.

Para el año 2017, la red de Cundinamarca se observó de menor tamaño y dividido en cuatro subredes, unidas por determinados actores. La principal causa asociada fue la rotación de integrantes en la red y el ingreso de asistentes técnicos agropecuarios del comité de cafeteros que prestaban asistencia en diferentes municipios lejanos. En ese sentido, se identificaron cinco actores clave, dos actores fuentes de información con las claves AT336 y el AT353, los cuales pertenecían a distintas zonas: el primero, de la Secretaría de Desarrollo Económico del municipio de Anapoima, y el segundo, de la Secretaría de Agricultura Departamental.

Por la estructura articulada de la red, con esta combinación de actores se lograba una cobertura $100 \%$ en la red para la difusión de información sobre el riesgo agroclimático, es decir, que estos actores por su posición podían hacer llegar la información a toda la red. Respecto a los actores colectores de información identificados fueron el AT335 y AT376, con la mayor cobertura en la red con $96.4 \%$. En Cundinamarca se observó que el AT336 no sólo tenía la función de proveedor de información sino también como articulador o intermediario en la red, sirviendo como enlace entre las tres subredes de las provincias del Tequendama, Gualivá y Valle de Ubaté. El AT336 junto con el AT349, alcanzaron $28.4 \%$ de fragmentación de los vínculos por su rol de nodo localregional o nacional.

\section{La Guajira}

La red de La Guajira en el año 2015 se apreció dividida en tres subredes, conectadas por dos importantes actores AT451 y AT459 de la organización Asohofrucol y con acción en los municipios Dibulla, Maicao, Riohacha y Sur de La Guajira, los cuales alcanzaron 100\% de cobertura como fuentes y buscadores de información en la red (Figura 4). Además, su índice de fragmentación era $53.7 \%$, lo que denotaba la importancia de incentivar su participación en la estrategia.

El primero de la Secretaría de Desarrollo Productivo, y el segundo, Asohofrucol, estos actores alcanzaron una cobertura $70 \%$ en la red para la difusión de información sobre el riesgo agroclimático. En el mismo sentido, se identificó al AT457 y AT459 como los actores buscadores de información con la máxima cobertura en red, con un porcentaje del 88.8\%. El AT457 junto a AT879 tuvieron el mayor índice de fragmentación en la red, con 32.1\%. Estos actores son parte de dos organizaciones reconocidas: Instituto Colombiano Agropecuario (ICA) y Fundación Cerrejón, las dos se localizan en el municipio de Uribia, por lo que se entiende su función de coordinar o enlazar a otros actores en la red. 

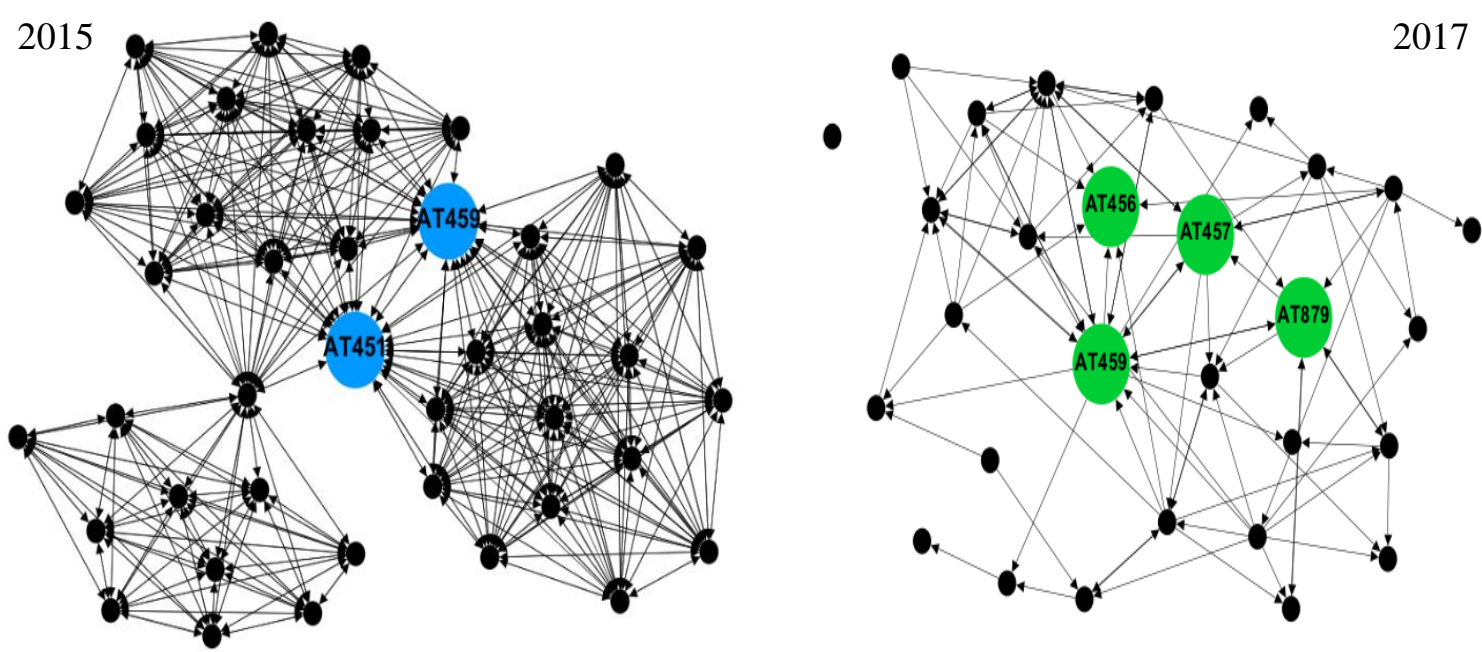

Figura 4. Red de confianza técnica de los ATA de La Guajira 2015 y 2017. Encuestas aplicadas a los ATA del proyecto MAPA, 2015 y 2017.

\section{Valle del Cauca}

La red Valle del Cauca en el año 2015, se identificaron cinco actores con una posición central, los actores con la clave AT832 y AT843 fueron las principales fuentes de información con una cobertura $89.7 \%$ en la red, el primero es parte del Instituto de educación técnica Profesional (Intep) del municipio Roldanillo, y el segundo, de la Corporación Colombiana de Investigación Agropecuaria (Figura 5). El AT815 y el AT817 fueron los actores colectores de información con la mayor cobertura con un porcentaje de $61.5 \%$, ya que su área de acción era diferente (Andalucía y Cali-Ginebra-Guacarí) y pertenecían a distintas organizaciones (Secretaria de Desarrollo de Andalucía y la Asociación comunitaria Asofrunidos de Ginebra).

Debido a la estructura de la red Valle del Cauca, los dos principales actores articuladores AT816 y AT843 obtuvieron un índice de fragmentación 43.7\%, es decir, si estos actores se excluían de la estrategia, la red podría tener una fuerte ruptura entre sus vínculos.
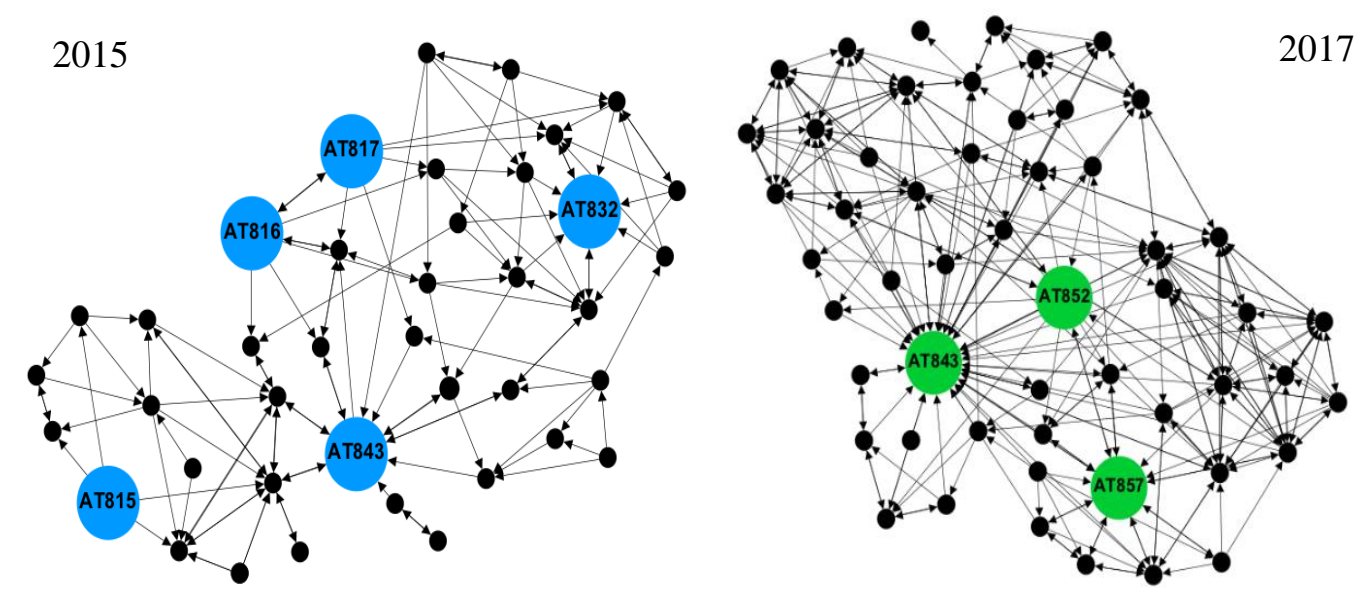

Figura 5. Red de confianza técnica de los ATA de Valle del Cauca 2015 y 2017. Encuestas aplicadas a los ATA del proyecto MAPA, 2015 y 2017. 
En el año 2017 la red de Valle del Cauca alcanzó una mayor integración, los actores articuladores AT843 y AT857, sólo llegaron a tener una cobertura de 21.3\%, lo que evidenció una menor fragilidad de la red. En el año 2017, el actor AT843 parte del proyecto MAPA, logró posicionarse en la red y sobresalió como principal fuente de información con una cobertura $98.1 \%$, así como, colector de información junto con el AT852 con una cobertura 92.2\%. Situación que favoreció a una mayor conectividad y una comunicación más directa con los asistentes técnicos del departamento.

En la red Valle del Cauca disminuyó significativamente $(p<0.05)$ el grado de intermediación de los actores (Cuadro 4). Por el contrario, el indicador de intermediación en la red de Cundinamarca y La Guajira aumentó de forma significativa $(p<0.05)$, lo cual evidencia que se construyeron una mayor cantidad de puentes o enlaces en las redes para favorecer la difusión de la información.

Cuadro 4. Comparación de medias de los indicadores de ego o nodo en las redes 2015 y 2017.

\begin{tabular}{cccccc}
\hline \multirow{2}{*}{ Indicador } & Departamento & Media & Media & Diferencia & Significancia \\
\hline Tamaño de la red nodal & Cundinamarca & 11.6 & 6.6 & -5 & 0 \\
& La Guajira & 15.1 & 5.4 & -9.7 & 0 \\
& Valle del Cauca & 4.6 & 8 & 3.4 & 0 \\
Intermediación $(\%)$ & Cundinamarca & 0.3 & 0.4 & 0.1 & 0 \\
& La Guajira & 0 & 0.7 & 0.7 & 0 \\
& Valle del Cauca & 0.7 & 0.6 & -0.1 & 0
\end{tabular}

Encuestas aplicadas a los ATA del proyecto MAPA, 2015 y 2017.

De esta manera, se podría afirmar que en los procesos de difusión del conocimiento es vital la presencia de agentes catalizadores u orquestadores para la gestión de las redes e impulsar todo el sistema, generando flujos de conocimiento, y una difusión eficaz del conocimiento (Muñoz y Santoyo, 2010). Para lograr la colaboración entre los miembros en este tipo de redes, es necesario la confianza, involucrar capacidades y competencias de los participantes y compartir normas, creencias y valores (Luna y Velasco, 2005).

\section{Conclusiones}

El análisis de redes sociales (ARS) permitió conocer las particularidades de cada una de las redes departamentales sirviendo de insumo para realizar una gestión del conocimiento diferenciada sobre el riesgo agroclimático, según el territorio y actores involucrados en los sistemas de producción agropecuarios. En este sentido, el análisis de las redes de confianza técnica de los asistentes técnicos agropecuarios en dos momentos de observación (al inicio y final del proyecto) permitió valorar los cambios ocurridos fruto de la estrategia intervención del proyecto MAPA para el fortalecimiento de la cultura agroclimática y mejorar la difusión de información.

En un entorno laboral inestable y en donde el rol de la asistencia técnica es prestado por diferentes actores, la gestión del conocimiento en redes de confianza técnica permitió generar un proceso de capacitación que tuvo como resultado un cambio en la percepción de los asistentes técnicos sobre la importancia del tema de riesgo agroclimático, como un tema transversal a la asistencia técnica. 
Así también, mejorar el posicionamiento de actores locales como coordinadores o animadores de su propia red para garantizar la sostenibilidad del proyecto y profundizar en su impacto en cada uno de los departamentos.

La gestión de la información por medio de las redes de confianza técnica entre los asistentes técnicos agropecuarios se mostró eficaz y eficiente en el uso de recursos, debido a la identificación de actores con mayor poder de difusión de la información y a la focalización para optimizar las relaciones de intercambio de información sobre el tema de riesgo agroclimático.

\section{Agradecimientos}

Los autores expresan su agradecimiento al proyecto 'Modelos de Adaptación y Prevención Agroclimática' financiado por el Fondo Adaptación y ejecutado por La Corporación Colombiana de Investigación Agropecuaria (Agrosavia). Así como, los asistentes técnicos de los departamentos de Cundinamarca, la Guajira y Valle del Cauca y a los profesionales de transferencia de tecnología del proyecto, quienes ayudaron en el levantamiento de la información y la gestión de las redes.

\section{Literatura citada}

Aguilar-Gallegos, N.; Martínez-González, E. G. y Aguilar-Ávila, J. 2017. Análisis de redes sociales: conceptos clave y cálculo de indicadores. Universidad Autónoma Chapingo (UACH)-Centro de Investigaciones Económicas, Sociales y Tecnológicas de la Agroindustria y la Agricultura Mundial (CIESTAAM). Serie: metodologías y herramientas para la investigación 5. Primera (Ed). Estado de México, México. 149 p. http://repositorio.chapingo.edu.mx:8080/bitstream/handle/20.500.12098/270/S-analisis-

17.pdf? sequence $=1 \&$ is Allowed $=\mathrm{y}$.

Aguilar-Gallegos, N.; Martínez-González, E. G.; Aguilar-Ávila, J.; Santoyo-Cortés, H.; MuñozRodríguez, M. y García-Sánchez, E. I. 2016. Análisis de redes sociales para catalizar la innovación agrícola: de los vínculos directos a la integración y radialidad. Colombia. Estudios Gerenciales. 140(32):197-207. Doi: https://doi.org/10.1016/j.estger.2016.06.006.

Bodin, Ö.; Crona, B. y Ernstson, H. 2017. Las redes sociales en la gestión de los recursos naturales: qué hay que aprender desde una perspectiva estructural. Redes. Rev. Hisp. Anális. Red Soci. 1(28):1-8. Doi: https://doi.org/10.5565/rev/redes.684.

Borgatti, S. P. 2006. Identifying sets of key players in a social network. Computational and mathematical organization theory. 12(1):21-34. Doi: https://doi.org/10.1007/s10588-0067084-x.

CIMMYT. 2019. Centro Internacional de Mejoramiento de Maíz y Trigo. Maíz para Colombia: visión 2030. E. Figueroa (Ed.). 28-29 pp. https://www.fenalce.org/archivos/maiz2030.pdf.

CEPAL. 2012. Comisión Económica para América Latina y el Caribe. Valoración de daños y pérdidas: Ola invernal en Colombia 2010-2011. Misión BID. Primera (Ed). Bogotá, Colombia. $247 \mathrm{p}$.

Corpoica. 2015a. Corporación Colombiana de Investigación Agropecuaria informe estudio línea de base del subsistema de asistencia técnica agropecuaria para los departamentos de Cesar, Cauca, Huila. La Guajira y Sucre. Colombia. Proyecto modelos de adaptación y prevención agroclimática-MAPA. 
Corpoica. 2015b. Corporación Colombiana de Investigación Agropecuaria. Informe caracterización del subsistema de asistencia técnica agropecuaria, para los departamentos de Boyacá, Córdoba, Cundinamarca, Magdalena, Santander, Tolima y Valle del Cauca. Colombia. Proyecto modelos de adaptación y prevención agroclimática-MAPA.

Landini, F. 2013. Perfil de los extensionistas rurales argentinos del sistema público. Argentina. Mundo Agrario. 27(14):1-23. https://www.mundoagrario.unlp.edu.ar/article/download/ MAv14n27a03/html_2?inline=1.

López, B. J.; Rendón, R.; Espinosa, T.; Torres, P. y Santellano, E. 2016. Medición de cobertura oculta en servicios de asistencia técnica y capacitación en el medio rural. Rev. Mex. Cienc. Agríc. 15(7):3089-3102.

Luna, M. y Velasco, J. L. 2005. Confianza y desempeño en las redes sociales. Rev. Mex. Sociol. 1(67):127-162.

Mayoral-García, M. B.; Cruz-Chávez, P. R.; Duarte-Osuna, J. de D. y Juárez-Mancilla, J. 2015. El perfil del extensionista rural en Baja California Sur (BCS), México. Rev. Glob. Negoc. 3(3):43-54. https://ssrn.com/abstract=2658324.

Méndez, M. J. 2015. Satisfacción con el empleo de extensionista rural: un estudio cualitativo en caldas. Colombia. Rev. Ceres. 62(3):241-250. Doi: http://doi.org/10.1590/0034737 X201562030003.

Monge, M. y Hartwich, F. 2008. Análisis de redes sociales aplicado al estudio de los procesos de innovación agrícola. Redes. Rev. Hisp. Anál. Red. Social. 2(14):1-31. Doi: https://doi.org/10.5565/rev/redes.118.

Monsalvo, A.; Jiménez, M. A.; García, J. L.; Sangerman-Jarquín, D. M.; Martínez, T. y Pimentel, J. L. 2017. Caracterización del perfil del extensionista rural en la zona oriente del Estado de México. Rev. Mex. Cienc. Agríc. 3(8):503-515. Doi: https://doi.org/10.29312/ remexca.v8i3.27.

Muñoz, M. y Santoyo, V. H. 2010. Pautas para desarrollar redes de innovación rural. In: del extensionismo agrícola a las redes de innovación rural. Santoyo, V. H. (Ed.). CIESTAAMUACH. Estado de México, México. 71-102 pp. Doi: https://doi.org/10.1017/ CBO9781107415324.004.

Pérez, Y. y Castañeda, M. 2009. Redes de conocimiento. Ciencias de la información. 1(40):3-20.

Powell, W. W. and Grodal, S. 2006. Networks of innovators. In: the oxford Handbook of innovation. Richard, R. N.; Mowery, D. C and Fagerberg, J. (Ed.). Oxford university press. USA. 56-85 pp.

Sáenz-Torres, S. M. 2014. Hacia la búsqueda de modelos alternativos de extensión rural en Colombia. México. Textual. 63(1):45-61. Doi: https://doi.org/10.5154/r.textual. 2014.63.003.

Schwaab, J. y Seibold, B. 2014. El potencial de las redes para el fortalecimiento de la sustentabilidad de la cooperación para el desarrollo. In: Fondos y redes globales. Reduciendo la brecha entre políticas globales y la implementación nacional. Lázaro, K. L.; Ayala, L. y Mueller, U. (Ed.). Instituto de Investigaciones. México, DF. 175-196 pp.

Vergara, W.; Rios, A. R.; Trapido, P. y Malarín, H. 2014. Agricultura y clima futuro en América Latina y el Caribe: impactos sistémicos y posibles respuestas. Banco interamericano de desarrollo. No. IDB-DP-329. 24 p.

Zarazúa-Escobar, J. A.; Almaguer-Vargas, G. y Rendón-Medel, R. 2012. Capital social. Caso red de innovación de maíz en Zamora, Michoacán, México. Cuadernos de desarrollo rural. 68(9):105-124. https://www.redalyc.org/articulo.oa?id=117/11723114006. 